\title{
Solve the Cubic Equation in a New Method with Engineering Application
}

\author{
Abdullah Dhayea Assi ${ }^{a}$ \\ ${ }^{a}$ Institute of Technology-Baghdad, Middle Technical University, Iraq-Baghdad
}

\section{ARTICLE INFO}

Article history:

Received 20 June 2020

Received in revised form 10 August 2020

Accepted 16 August 2020

\section{Keywords:}

Matrix tensor

Eigen values

Eigen vectors

Roots

Cubic equation

Principal angle

\begin{abstract}
A B S T R A C T
Up to date the cubic equation or matrix tensor is consisting of nine values such as stress tensor that turns into the cubic equation which has been used for solving classic method. This is to impose an initial root several times to get it when achieves the equation and any other party is zero. Then dividing the cubic equation on the equation of the root. After that dividing the cubic equation on the equation of the root and using the classical method to find the rest of the roots. This is a very difficult issue, especially if the roots are secret or large for those who are looking in a difficult field or even for those who are in the examination room. In this research, two equations were reached, one that calculates the angle and the other that calculates the three roots at high accuracy without any significant error rate. By taking advantage of the traditional method, not by imposing a value to get the root of that equation, but by imposing an equation to get the solution equation that gives the value of that root. After imposing that equation, the general equation was derived from which that calculated the three roots directly and without any attempts. The angle that was implicitly derived during the derive of the main equation is calculated by taking advantage of the constants that do not change (invariants) for the matrix tensor (T).
\end{abstract}

\section{Introduction:}

The main problem in engineering calculations is to determine the Eigen Values of the matrix (T), which is called matrix tensor $(T))$. In general, if the $(n * n)$ are the dimensions of the matrix $(T)$, it has $(n)$ of Eigen values and (n) of Eigen vectors, and this needs to be done manually. In order to increase the accuracy, the computer usually uses to find out all the solutions required for the matrix (T). In other words, finding all or most of the roots and vectors is not mainly depend on the form of the matrix (T), as much as it depends on what the required solutions are. In particular, the Eigen values of the matrix extended (Matrix Tensor) is calculated to keep $(3 * 3)$, and this matrix, which consists of nine components are inherently transformed into an equation of the third degree, (equation cubic). By obtaining eigenvalues, the roots

* Corresponding author.

E-mail address: drabdullah_dhayea@mtu.edu.iq (Abdullah Dhayea Assi) of the cubic equation became research achievement as well as being mathematically engineered achievement. Fifty years ago, as well as the generations that followed mathematicians, physics, and astronomy have tried to solve the cubic equation, but all formulas were raised worse than traditional formula. The first scientist who solved the cubic equation is del Ferro (Scipione del Ferro (1465-1626)) in the early sixteenth century, where he lectured in mathematics and engineering at the University of Bologna from 1496 until 1526. He wrote a solution of cubic equation in the manuscript, which passed to his pupil Antonio Maria Fiore (Antonio Maria Fior (Florido)). There are no reports of deployment of such a manuscript and was racing to find a solution with Niccolo Fontana Tataaelia (Nicol `o Fontana Tartaglia (1500-1557)) Kumar et al. [1], who led to the recent discovery of a way to solve the cubic equation of a certain type. Girolamo Cardano (1501-1576) was able to convince him 
to dissolve it with a full explanation after the swearing in front of Niccolo Fontana to keep it a secret. He found a solution for the news published in the newspapers and saw that Cardano published free of charge, and this is what he did in Ars Magna in 1545. However, Cardano went further than his predecessors because it is summed up nearly 13 formulae and share it one after the death of Niccolo Fontana Tataaelia. Cardano scholar athlete was a physician and astronomer, and he had able to publish this formula in his book $1545 \mathrm{AD}$. His method required the following Karunia et al. [2]:

\subsection{Cardano formula Karunia et al. [2]}

First, simplify the equation to become standard in the following form:

$$
x^{3}+a x^{2}+b x+c=0
$$

Then get rid of the coefficient of the second division with appropriate compensation $\mathrm{p}, \mathrm{q}, \mathrm{t}$ to become the new form of the equation:

$$
t^{3}+p t+q=0
$$

where:

$$
p=b-\frac{a^{2}}{3} \quad \text { and } \quad q=c+\frac{2 a^{3}-9 a b}{27}
$$

And appropriate compensation in equation (2), it can be obtained:

$$
u^{3}+v^{3}+(3 u v+p)(u+v)+q=0
$$

Here, Cardano supposed a new limit for variables u and v so:

$$
3 u v+p=0
$$

When this merges in Equ. (3) by compensating $\mathrm{v}$ we get:

$$
u^{6}+q u^{3}-\frac{p^{3}}{27}=0
$$

It can be seen that this equation of the sixth grade that can simplify into the second division in $\mathrm{u}^{3}$ and resolved to become directly:

$$
u^{3}=-\frac{q}{2} \pm \sqrt{\frac{q^{2}}{4}+\frac{p^{3}}{27}}
$$

Based on that, u can be calculated by Eqn. 7 as:

$$
u=\sqrt[3]{-\frac{q}{2} \pm \sqrt{\frac{q^{2}}{4}+\frac{p^{3}}{27}}}
$$

Since $\mathrm{t}=\mathrm{v}+\mathrm{u}, \mathrm{t}=\mathrm{x}+\mathrm{a} / 3$, and $\mathrm{v}==-\mathrm{p} / 3 \mathrm{u}$, we find that:

$$
x=-\frac{p}{3 u}+u-\frac{a}{3}
$$

Note that there are 6 roots to calculate the likelihood of $u$ in Equ. (7), because the square root of two possibilities holds (+ and -) for three roots. But, the square root does not affect the resulting value of $t$ (However, one should pay any attention to three cases to avoid division by zero):

First: If $\mathrm{p}=\mathrm{q}=0$, then $\mathrm{u}$ has three real roots.

Second: If $\mathrm{p}=0$ and $\mathrm{q} \neq 0$, the:

Third: If the $\mathrm{p} \neq 0$ and $\mathrm{q}=0$ is:

$$
u=0 \quad \text { and } \quad v=-\sqrt[3]{q}
$$

$$
u=\sqrt{\frac{p}{3}} \quad \text { and } \quad v=-\sqrt{\frac{p}{3}}
$$

In any of the three cases, the roots are:

$t=u+v=0, \quad t=w_{1} u-\frac{p}{3 w_{1} u}=\sqrt{-p}$,

$t=\frac{u}{w_{1}}-\frac{w_{1} p}{3 u}=-\sqrt{-p}$

where:

$w_{1}=e^{i \frac{2 \pi}{3}}=-\frac{1}{2}+i \frac{\sqrt{3}}{2}$

This way in this time is more complex than the conventional formula. Which are summarized in the following classic method:

\subsection{Classic Method:}

Solving the following cubic equation using the Classic Method $[3,4]$ :

$$
\lambda^{3}-5 * \lambda+2=0
$$

The solution by using this method requires the imposition of a preliminary investigating of the root of the equation through several attempts:

$$
\begin{gathered}
\lambda^{3}-5 \lambda+2=0 \quad \text { let } \lambda_{1}=2 \Rightarrow 8-5(2)+2=0 \quad \text { ok } \\
\therefore \frac{\lambda^{3}-5 \lambda-2}{\lambda-2}=\lambda^{2}+2 \lambda-1 \Rightarrow \therefore \lambda^{2}+2 \lambda-1=0 \\
\lambda=\frac{-b \pm \sqrt{b^{2}-4 a c}}{2 a} \Rightarrow a=1, b=2, c=-1 \\
\therefore \lambda=\frac{-(2)}{2(1)} \pm \frac{\sqrt{(2)^{2}-4(1)(-1)}}{1(2)}=-1 \pm \sqrt{2} \\
\lambda_{2}=-1-\sqrt{2}=-2.42, \quad \lambda_{3}=-1+\sqrt{2}=0.42 \\
\text { and }: \lambda_{1}=2
\end{gathered}
$$

\section{The Theoretical Part:}

Equ. (9) below, which form the equivalent of a limited class (n) for any form of multi-limits in $(\lambda)$ have a $(n)$ of the roots $(n \lambda 2, \ldots \ldots . .1 \lambda$ (not necessarily different Ghali et al. [5].

$$
|A-\lambda I|=\left|\begin{array}{ccc}
a_{11}-\lambda & a_{12} & a_{1 n} \\
a_{21} & a_{22-} \lambda & a_{2 n} \\
a_{n 1} & a_{n 2} & a_{n n-} \lambda
\end{array}\right|
$$

where this equation is called a characteristic equation or matrix characteristic. Given these roots, there are values for the vertical vector(v) obtained from the following equation:

$$
|A-\lambda I| V=0
$$

Before explaining how to test the property of Equ. (10) that lead to Equ. (1), Equ. (1) can be written as the following:

$$
\mathrm{AV}=-\lambda \mathrm{V}
$$

On the left side of the equation, (A) is a matrix of $(n * n)$ dimensions, and vector $(\mathrm{V})$ is a vector of $n$-vertical dimensions. The question that arises, of course, is the meaning of product $\mathrm{A} * \mathrm{~V}$. It is clear that the AV is the vector as well. Accordingly, matrix (A) can therefore be considered effective or transformational when it affects a V, which produces a new $\mathrm{n}$-dimensional vector. This transformation in the space 
of all $\mathrm{n}$ of deportation can be realized more clearly if $\mathrm{n}=3$. For example, in such a situation, one can imagine a matrix $\mathrm{A}\left(3^{*} 3\right)$ as affecting the vector three-dimensional $\mathrm{V}$ and turns it one way or another into three dimensional vector. In three dimensions, In the three dimensions, such a transformation can be conceived as involving a vector rotation, prolonging the vector or shrink, or possibly both, of the overall situation, where $\mathrm{n}>3$ we can imagine A rotation or possibly prolong vector or reduced in a vacuum -dimensional $\mathrm{n}$, though the such a perception is not as simple as in the case $\mathrm{n}=3$.

Now, many researches on the theory of matrices have been completed in Germany, so the word Aiken "eigen" means in German " self or true ". Therefore, the values of $\lambda$ and vectors corresponding $\mathrm{V}$ became known values of self (eigen values) and (eigen vectors) sequentially, and this term will be used in the same equation (9) to find the values of $\lambda$. Here are some observations that should be displayed.

\subsection{Methods of Numerical Analysis to Accelerate the Convergence:}

To illustrate the basic idea of numerical methods to analyse the acceleration of convergence and to find values and eigenvectors. Suppose that Javier et al. [6]:

$$
A \vec{Y}^{(i)}=\lambda_{i} \vec{Y}^{(i)}
$$

The terms of: -

A is a symmetric matrix that wants to find their roots.

$\vec{Y}^{(i)}$ is the eigenvectors which is characteristic.

$\lambda_{\mathrm{i}}$ is the characteristic eigenvalues,

where $i=1,2,3, \ldots \ldots \ldots \ldots . . . . n$.

Since that is a set of orthogonal bases, then we can use any name and optional vector $\mathrm{p}$ as a linear combination of the vector $\vec{Y}^{(i)}$, as shown in the following:

$$
p=c_{1} \overrightarrow{\mathrm{Y}}^{(1)}+c_{2} \overrightarrow{\mathrm{Y}}^{(2)}+\ldots \ldots \ldots+c_{n} \overrightarrow{\mathrm{Y}}^{(n)}
$$

And multiplying the equation (5) in the symmetric matrix A, we get:

$$
A \vec{p}=\lambda_{1} c_{1} \overrightarrow{\mathrm{Y}}^{(1)}+\lambda_{2} c_{2} \overrightarrow{\mathrm{Y}}^{(2)}+\ldots . .+\lambda_{n} c_{n} \overrightarrow{\mathrm{Y}}^{(n)}
$$

Assume that the root $\lambda 1$ for the largest coefficient is real and that the root:

Where: $r=1,2,3, \ldots \ldots \ldots ., n$

$$
\left|\lambda_{r}\right| \neq\left|\lambda_{1}\right|
$$

Because we care about the real root $\lambda 1$ if we re-formulate the equation (14) as follows for $\lambda_{1}$ :

$$
A \vec{P}=\lambda_{1}\left[C_{1} \overrightarrow{\mathrm{Y}}^{(1)}+\left(\frac{\lambda_{2}}{\lambda_{1}}\right) C_{2} \overrightarrow{\mathrm{Y}}^{(2)}+\ldots \ldots+\left(\frac{\lambda_{n}}{\lambda_{1}}\right) C_{n} \overrightarrow{\mathrm{Y}}^{(n)}\right]
$$

And multiplying the equation (6) by A matrix to $\mathrm{m}$ times and reformulated as in the equation (7), we get the following form:

$\mathrm{A}^{(m)} \vec{p}=\lambda_{1}^{m}\left[C_{1} \overrightarrow{\mathrm{Y}}^{(1)}+\left(\frac{\lambda_{2}}{\lambda_{1}}\right)^{2} C_{2} \overrightarrow{\mathrm{Y}}^{(2)}+\ldots . .+\left(\frac{\lambda_{m}}{\lambda_{1}}\right)^{m} C_{n} \overrightarrow{\mathrm{Y}}^{(m)}\right]$

And

multiplying the equation (16) in the matrix A, we get the following form:

$$
\mathrm{A}^{m+1} \vec{p}=\lambda_{1}^{m+1}\left[C_{1} \overrightarrow{\mathrm{Y}}^{(1)}+\left(\frac{\lambda_{2}}{\lambda_{1}}\right)^{m+1} C_{2} \overrightarrow{\mathrm{Y}}^{(2)}+\ldots . .+\left(\frac{\lambda_{n}}{\lambda_{1}}\right)^{m+1}+C_{n} \overrightarrow{\mathrm{Y}}^{(n)}\right]
$$

suppose that:

So:

$$
\left|\lambda_{1}\right|>\left|\lambda_{2}\right|>\ldots \ldots .>\left|\lambda_{n}\right|
$$

$$
\lim _{m \rightarrow \infty}\left(\frac{\lambda_{1}}{\lambda_{2}}\right)^{m}=0
$$

If $\mathrm{C}_{1}$ is not equal to zero and that the value of vector elements $\mathrm{p}$ relatively large, $\lambda_{1}$ can extract value from equations (16) and (17) in the following form:

$$
\lim _{n \rightarrow \infty}\left(\frac{A^{m+1} \vec{P}}{A^{m} \vec{P}}\right)=\left(\frac{\lambda_{1}^{m+1} C_{Y^{\prime}} \vec{Y}^{(1)}}{\lambda_{1}^{m} C_{1} \vec{Y}^{(1)}}\right)=\lambda_{1}
$$

Applying the equation (18) we need to get a polynomial equation, and in the case multiply the vector chosen $\mathrm{p}$ in the matrix symmetric. We get a new vector and multiplying this vector in the matrix corresponding A after extracting the greatest value from the values of its elements, which represent $\lambda_{1}$ and the division of all elements on these values $\left(\lambda_{1}\right)$. As a result of the multiplying, we get a new vector and make it the same previous steps and repeating this process, we get the serial number of unspecified vectors $\mathrm{Y}$, as explains it in the following:

$$
Y_{i+1}=A Y_{i}=A^{i+1} Y_{0}
$$

In the case of the convergence of the values of the vector elements $\mathrm{Yi}$ with the values of the vector elements $\mathrm{Y}_{\mathrm{i}}+1 \mathrm{E}$ ratio $\varepsilon=\mathrm{Yi}$ / $\mathrm{Yi}+1$ in this case we get the value of the desired root $\left(\lambda_{1}\right)$ as the convergence does not depend on the choice of $\mathrm{y}_{0}$.

\subsection{Repeated Roots}

In the case of different eigenvalues from each other and that the foundations of eigenvectors perpendicular. Assume that $\mathrm{X}$ is the vector that we got after the $\mathrm{K}$ of repetition Javier et al. [6]:

$$
\vec{X}=C_{1} V_{1}+C_{2}\left(\frac{\lambda_{2}}{\lambda_{1}}\right)^{k} V_{2}+\ldots \ldots \ldots+C_{n}\left(\frac{\lambda_{n}}{\lambda_{1}}\right)^{k} V_{n}
$$

We suppose that:

$$
\Sigma_{n}=C_{n}\left(\frac{\lambda_{n}}{\lambda_{1}}\right)^{k}
$$

So:

$$
\vec{X}=C_{1} V_{1}+\Sigma_{2} V_{2}+\ldots \ldots \ldots \ldots \ldots+\Sigma_{n} V_{n}
$$

We assume that the vector $\mathrm{V}_{\mathrm{i}}$ is the (normalized), so: $A X=C_{1} \lambda_{1} V_{1}+\Sigma_{2} \lambda_{2} X_{2} \ldots \ldots+\Sigma_{n} \lambda_{n} V_{n}$

and: $\mathrm{X}^{t} \mathrm{AX}=\lambda_{1}\left|C_{1}\right|^{2}+\lambda_{2}\left|\Sigma_{2}\right|^{2}+\ldots . .+\lambda_{n}\left|\Sigma_{n}\right|^{2}$

Also: $\mathrm{X}^{t} \mathrm{X}=\left|C_{1}\right|^{2}+\left|\Sigma_{2}\right|^{2}+\ldots \ldots \ldots \ldots+\left|\Sigma_{n}\right|^{2}$

When repeat $\mathrm{k}$ increases, each $\Sigma_{\mathrm{i}}$ close to zero, and this way we get Riley (Rayleigh equation), Which is: $\frac{\mathrm{X}^{t} \mathrm{AX}}{\mathrm{X}^{t} \mathrm{X}}$

$$
\overline{X^{t} X}
$$

\subsection{Shift of origin Salas et al. [4]: -}

The problems and difficulties that also appear when using the methods of numerical analysis in some arrays are not getting the convergence required for the values of self-eigen values of the matrix (A). In this case, we need to shift to be sufficient for the values of selfeigen values of the matrix $A$ and the same value selected. Let $S$ and subtracting this value from the matrix elements of diagonal and will be represented mathematically as follows:

$$
\begin{aligned}
& \mathrm{AX}=\lambda \mathrm{X}=(\lambda-S) \mathrm{X}+S \mathrm{X} \\
& \Rightarrow(\mathrm{A}-S I) \mathrm{X}=(\lambda-S) \mathrm{X}
\end{aligned}
$$

Equation (26) can be written in the following form:

$$
B X=\eta \mathrm{X}
$$

where: $(\mathrm{A}-S I)=B$ and $(\lambda-S)=\eta$

Using this method, the eigenvalues will change while eigenvectors are not changed at all and that the ratio of convergence depends on $\left(\lambda_{n}\right.$ $\left.+1 / \lambda_{n}\right)$ and if we apply this method to the new matrix (A-SI), the eigenvalues of the new matrix is a $\left(\lambda_{\mathrm{i}}-\mathrm{S}\right)$. In this case, the percentage of 
convergence depends on: $\frac{\lambda_{2}-S}{\lambda_{1}-S}$

\subsection{Convergence Method in the Case of $\left(\lambda_{2}=-\lambda_{1}\right)$}

This method is used to solve the third problem and where real roots equal in value and different in the signal and if we use the previous methods for finding eigenvalues of the matrix $\mathrm{A}$, we do not get the convergence. To illustrate the idea mathematically Suppose that the vector $\vec{Y}^{(p)}$ is a linear combination for two of the eigenvectors corresponding to the real roots, and the mathematical relationship will be as follows Gao et al. [8]:

$$
Y^{p+2}=\lambda_{2} Y^{p}
$$

The proportion of convergence is $\left(Y_{i}^{p+2} / Y_{i}^{p}\right)$, and it will stabilize at a constant value $\lambda$, and to get rid of this case, we use the displacement method at the center (shift of origin) and so we get the desired convergence.

\section{The derivation of the new method to solve cubic equations.}

The aim of the research or the basic requirement is to solve the cubic equation by several attempts to get constant roots instead of solving each formula of formulas for each case (Use of attempts to obtain the equations of the roots and not on the amounts of roots). The required cubic equation can be solved as follows:

$$
\begin{gathered}
x^{3}+\mathrm{A} x^{2}+B x+C=0 \\
\text { *If } \quad K x=K y+A, \text { let } \quad K=3 \\
\therefore 3 x=3 y+A \Rightarrow y=x-\frac{A}{3}
\end{gathered}
$$

Compensating in the basic equation above:

$$
\begin{aligned}
& \left(y-\frac{\mathrm{A}}{3}\right)^{3}+\mathrm{A}\left(y-\frac{\mathrm{A}}{3}\right)^{2}+\mathrm{B}\left(y-\frac{\mathrm{A}}{3}\right)+C=o \\
& y^{3}-3 y^{2}\left(\frac{\mathrm{A}}{3}\right)+3 y\left(\frac{\mathrm{A}}{3}\right)^{2}-\left(\frac{\mathrm{A}}{3}\right)^{3}+\mathrm{A}\left(y^{2}-2 y\left(\frac{\mathrm{A}}{3}\right)+\frac{\mathrm{A}^{2}}{g}\right)+\mathrm{B} y-\frac{\mathrm{BA}}{3}+C=0 \\
& \therefore y^{3}-\mathrm{A} y^{2}+\frac{3 y \mathrm{~A}^{2}}{9}-\frac{\mathrm{A}^{3}}{27}+\mathrm{A} y^{2}-\frac{2 \mathrm{~A}^{2}}{3} y+\frac{\mathrm{A}^{3}}{9}+\mathrm{B} y-\frac{\mathrm{BA}}{3}+C=0 \\
& \therefore y^{3}+\left(\mathrm{B}-\frac{\mathrm{A}^{2}}{3}\right) y-\left(-\frac{2 \mathrm{~A}^{3}}{27}+\frac{\mathrm{BA}}{3}-C\right)=0 \quad \therefore y^{3}+\kappa_{1} y+\kappa_{2}=0
\end{aligned}
$$

Where $\kappa_{1}=\mathrm{B}-\frac{\mathrm{A}^{2}}{3}=\frac{3 \mathrm{~B}-\mathrm{A}^{2}}{3}$ and

$$
\kappa_{2}=-\frac{2 \mathrm{~A}^{3}}{27}+\frac{\mathrm{BA}}{3}-C=\frac{2}{27}\left(-\mathrm{A}^{3}+4.5 \mathrm{BA}-13.5 C\right)
$$

Using the laws of trigonometry, as follows:

$$
\begin{aligned}
& 4 \cos ^{3} \alpha=3 \cos \alpha+\cos 3 \alpha \Rightarrow \cos ^{3} \alpha=\frac{3}{4} \cos \alpha=\frac{1}{4} \cos 3 \alpha \\
& \therefore \cos ^{3} \alpha-\frac{3}{4} \cos \alpha-\frac{1}{4} \cos 3 \alpha=0 \\
& \text { if } \quad U=\cos \alpha \quad \therefore U^{3}-\frac{3}{4} U-\frac{1}{4} \cos 3 \alpha=0
\end{aligned}
$$

$$
\begin{aligned}
& U^{3}-\frac{3}{4} U=\frac{1}{4} \cos 3 \alpha \Rightarrow \therefore 4 U^{3}-3 U=\cos 3 \alpha \\
& \text { If } \quad \alpha=60 \Rightarrow U=\cos 60=0.5 \Rightarrow 0.5-3(0.5)=\cos 3 \alpha \\
& -1=\cos 3 \alpha \quad \Rightarrow \cos 3 \alpha=-1
\end{aligned}
$$

Then:

$$
\begin{array}{lll}
\text { If } & \alpha_{1}=60 \Rightarrow \cos 3(60)=-1 \quad \text { satisfy } & \\
\text { If } & \alpha_{2}=\alpha_{1}+120 \Rightarrow \cos 3(60+120)=-1 & \text { satisfy } \\
\text { If } & \alpha_{3}=\alpha_{1}+240 \Rightarrow \cos 3(60+240)=-1 & \text { satisfy }
\end{array}
$$

This means that the cubic equation that is three roots obtained by three angles $(\alpha, 120+\alpha, 240+\alpha)$ each root of these roots must be at an angle from these angles, which does not mean that the roots repeat the same final value as it may be repeated or not repeated depending on the specificity of the equation or matrix tensor that will become an equation. So:

$$
\begin{aligned}
& \therefore U^{3}-\frac{3}{4} U-\frac{1}{4} \operatorname{Cos} 3 \alpha=0 \\
& y^{3}+k_{1} y+k_{2}=0 \\
& \text { let } \quad y=V U \\
& \therefore V^{3} U^{3}+k_{1} V U+k_{2}=0 \Rightarrow \\
& U^{3}+\frac{k_{1}}{V^{2}} U+\frac{k_{2}}{V^{3}}=0
\end{aligned}
$$

In order to make equations (31) and (33) equal each other, the following conditions must be satisfied:

$$
\begin{aligned}
& \text { (1) } \quad \frac{k_{1}}{V^{2}}=-\frac{3}{4} \quad \text { and (2) } \quad \frac{k_{2}}{V^{3}}=-\frac{1}{4} \cos 3 \alpha \\
& \therefore \text { from }(1) \Rightarrow V=\sqrt{\frac{-4 k_{1}}{3}} \text { and } \\
& \text { from (2) } \Rightarrow \cos 3 \alpha=\frac{-4 k_{2}}{V^{3}} \text {. } \\
& \text { sub. } V \quad \text { in equation(34) we get : } \\
& \cos 3 \alpha=\frac{-4 k_{2}}{V^{3}}=\frac{-4 k_{2}}{\left(\frac{-4 k_{1}}{3}\right)^{3 / 2}}
\end{aligned}
$$

sub. $k_{1} \& k_{2}$ in equation(e) we get:

$$
\begin{aligned}
& \therefore \cos 3 \alpha=\frac{-4\left[\frac{2}{27}\left(-A^{3}+4.5 A B-13.5 C\right)\right]}{\left[-\frac{4}{3}\left(\frac{3 B-A^{2}}{3}\right)\right]^{3 / 2}} \\
& =\frac{-\frac{8}{27}\left[-A^{3}+4.5 A B-13.5 C\right]}{\left[\frac{4}{9}\left[3 B-A^{2}\right]\right]^{3 / 2}} \\
& \therefore \cos 3 \alpha=\frac{-\frac{8}{27}\left[-A^{3}+4.5 A B-13.5 C\right]}{\frac{8}{27}\left[3 B-A^{2}\right]^{3 / 2}} \Rightarrow \\
& \therefore \cos 3 \alpha=\frac{\left[A^{3}-4.5 A B+13.5 C\right]}{\left[3 B-A^{2}\right]^{3 / 2}}
\end{aligned}
$$

Where it was to get rid of the negative after inserted into the equation: 
Let $\quad \theta=3 \alpha \Rightarrow \alpha=\frac{\theta}{3}$

$\therefore \quad \cos \theta=\frac{\left[A^{3}-4.5 A B+13.5 C\right]}{\left[A^{2}-3 B\right]^{1.5}}$

$\therefore \theta_{1}=\alpha=\frac{\theta}{3}, \quad \theta_{2}=\alpha+120=\frac{\theta}{3}+120$

$\theta_{3}=\alpha+240=\frac{\theta}{3}+240$

To get the greatest value:

$\therefore y_{1}$ at $\theta_{1} \Rightarrow y_{1}=\sqrt{\frac{A^{2}-3 B}{9}} * \cos \left(\theta_{1}\right)$

$\therefore y_{2}$ at $\theta_{2} \Rightarrow y_{2}=\sqrt{\frac{A^{2}-3 B}{9}} * \cos \left(\theta_{2}\right)$

$\therefore y_{3}$ at $\quad \theta_{3} \Rightarrow y_{3}=\sqrt{\frac{A^{2}-3 B}{9}} * \cos \left(\theta_{3}\right)$

But what is needed is ( $\mathrm{x})$ and not $(\mathrm{y})$ because the general equation is a function of $(\mathrm{x})$ either $(\mathrm{y})$ is a dummy variable or default.

$$
\begin{aligned}
& \because \quad x=y+\frac{A}{3} \Rightarrow \\
& \therefore x_{1}=\sqrt{\frac{A^{2}-3 B}{9}} * \cos \left(\frac{\theta}{3}\right)+\left(\frac{A}{3}\right) \\
& x_{2}=\sqrt{\frac{A^{2}-3 B}{9}} * \cos \left(\frac{\theta}{3}+120\right)+\left(\frac{A}{3}\right) \\
& \& x_{3}=\sqrt{\frac{A^{2}-3 B}{9}} * \cos \left(\frac{\theta}{3}+240\right)+\left(\frac{A}{3}\right) \\
& \because \theta_{1}=\frac{\theta}{3}, \quad \theta_{2}=\frac{\theta}{3}+120, \quad \theta_{3}=\frac{\theta}{3}+240 \\
& \therefore \quad x_{1}=\sqrt{\frac{A^{2}-3 B}{9}} * \cos \left(\theta_{1}\right)+\left(\frac{A}{3}\right) \\
& x_{2}=\sqrt{\frac{A^{2}-3 B}{9} * \cos \left(\theta_{2}\right)+\left(\frac{A}{3}\right)} \\
& \& \quad x_{3}=\sqrt{\frac{A^{2}-3 B}{9}} * \cos \left(\theta_{3}\right)+\left(\frac{A}{3}\right)
\end{aligned}
$$

If these equations are applied, the real roots of $(x)$ is not given because the first hypothesis, that has been imposed $(3 x$ $=3 \mathrm{y}+\mathrm{A}$ ), based on a fixed proposal and is not real. To find a real constant any $(\mathrm{Kx}=\mathrm{Ky}+\mathrm{A})$. It should multiply the amounts in (K).

Then:

$$
\begin{gathered}
x_{i}=K * \sqrt{\frac{A^{2}-3 B}{9}} * \cos \left(\theta_{i}\right)+\frac{A}{3} \\
\text { where } i=1,2 \& 3
\end{gathered}
$$

Or

$$
x_{1}=K * \sqrt{\frac{A^{2}-3 B}{9}} * \cos \left(\theta_{1}\right)+\frac{A}{3}
$$

$$
x_{2}=K * \sqrt{\frac{A^{2}-3 B}{9}} * \cos \left(\theta_{2}\right)+\frac{A}{3}
$$

$$
x_{3}=K * \sqrt{\frac{A^{2}-3 B}{9}} * \cos \left(\theta_{3}\right)+\frac{A}{3}
$$

Using attempts and experience for all kinds of Tensor, $\mathrm{K}$ is calculated. And all kinds of problems gave one value of $(\mathrm{K})$ and the truth here is no possibility for error because the three roots should be given the same value of $(\mathrm{K})$ and this ensures the correctness:

Take unresolved issues ((i.e. the process inversed our solution and the equation wanted)).

We have the roots $\left(x_{1}=3, x_{2}=1, x_{3}=2\right)$ for the following cubic equation:

$$
x^{3}-6 x^{2}+11 x-6=0
$$

This means that $(A=6, B=11, C=6)$ and the equation of the angle $\theta$ we calculate the new value:

$$
\begin{aligned}
& \cos \theta=\frac{\left[A^{3}-4.5 A B+13.5 C\right]}{\left[A^{2}-3 B\right]^{1.5}} \Rightarrow \\
& \theta=\cos ^{-1}\left[\frac{\left[A^{3}-4.5 A B+13.5 C\right.}{\left[A^{2}-3 B\right]^{1.5}}\right] \\
& \theta=\cos ^{-1}\left[\frac{\left[A^{3}-4.5 A B+13.5 C\right]}{\left[A^{2}-3 B\right]^{1.5}}\right] \\
& =\cos ^{-1}\left[\frac{\left[6^{3}-4.5 * 6 * 11+13.5 * 6\right]}{\left[6^{2}-3 * 11\right]^{1.5}}\right]=90^{\circ} \\
& \because \theta_{1}=\frac{\theta}{3}=30^{\circ}, \quad \theta_{2}=\frac{\theta}{3}+120=150^{\circ} \\
& \theta_{3}=\frac{\theta}{3}+240=270^{\circ} \\
& 3=K * \sqrt{\frac{6^{2}-3 * 11}{9}} * \cos (30)+\frac{6}{3} \Rightarrow K=2 \\
& 1=K * \sqrt{\frac{6^{2}-3 * 11}{9}} * \cos (150)+\frac{6}{3} \Rightarrow K=2 \\
& 2=K * \sqrt{\frac{6^{2}-3 * 11}{9}} * \cos (270)+\frac{6}{3} \Rightarrow K=2 \\
& \therefore x_{1}=2 * \frac{1}{3}\left(A^{2}-3 B\right)^{0.5} * \cos \left(\theta_{1}\right)+\frac{A}{3} \\
& x_{2}=2 * \frac{1}{3}\left(A^{2}-3 B\right)^{0.5} * \cos \left(\theta_{2}\right)+\frac{A}{3} \\
& x_{3}=2 * \frac{1}{3}\left(A^{2}-3 B\right)^{0.5} * \cos \left(\theta_{3}\right)+\frac{A}{3}
\end{aligned}
$$

To prove that the extended matrix transformed into a cubic equation, as well as identifying signals cubic equation:

If we assume that our values are established as a Tensor, as follows Govindan et al. [9]: -

$$
[T]=\left|\begin{array}{lll}
t_{11} & t_{12} & t_{13} \\
t_{21} & t_{22} & t_{23} \\
t_{31} & t_{32} & t_{33}
\end{array}\right|
$$

This matrix can be converted from one format to format arrays equations where the resulting equation of the third degree, which are as follows: $\lambda^{3}+\mathbf{A} \lambda^{2}+\mathbf{B} \lambda+\mathbf{C}=\mathbf{0}$

The constants in this equation can be calculated using the equation feature $|\mathrm{T}-\lambda \mathrm{I}|$, namely:

$$
|T-\lambda I|=\left|\begin{array}{ccc}
t_{11}-\lambda_{i} & t_{12} & t_{13} \\
t_{21} & t_{22}-\lambda_{i} & t_{23} \\
t_{31} & t_{32} & t_{33}-\lambda_{i}
\end{array}\right|
$$

Therefore, these constants can be taken the following forms: $A=\left(t_{11}+t_{22}+t_{33}\right)=$ Trace[T]

(47a) 
$\mathrm{B}=\left[\mathrm{t}_{22} * \mathrm{t}_{11}+\mathrm{t}_{33} * \mathrm{t}_{11}+\mathrm{t}_{22} * \mathrm{t}_{33}+\mathrm{t}_{23} * \mathrm{t}_{32}-\mathrm{t}_{32} * \mathrm{t}_{21}-\mathrm{t}_{13} * \mathrm{t}_{31}\right]$

$=\Sigma$ miner $[\mathrm{T}]$ (47b)

$\mathrm{C}=\left[\mathrm{t}_{11} * \mathrm{t}_{22} * \mathrm{t}_{33}-\mathrm{t}_{11} * \mathrm{t}_{23} * \mathrm{t}_{32}-\mathrm{t}_{12} * \mathrm{t}_{21} * \mathrm{t}_{33}+\mathrm{t}_{12} * \mathrm{t}_{23} * \mathrm{t}_{31}\right.$

$\left.+t_{13} * t_{21} * t_{32}-t_{22} * t_{31} * t_{13}\right]=\operatorname{det}[\mathbf{T}] \quad$ (47ca)

The cubic equation can be written by the mathematical formula, i.e. use $\mathrm{X}$ as follows:

$X^{3}-A X^{2}+B X-C=0$

$\therefore \theta=\cos ^{-1}\left[\frac{A^{3}-4.5 A B+13.5 C}{\left(A^{2}-3 B\right)^{1.5}}\right]$

$X_{1}=\frac{1}{3}\left[2\left(A^{2}-3 B\right)^{0.5} * \cos \left(\theta_{1}\right)+A\right]$

where $\theta_{1}=\frac{\theta}{3}$

$X_{2}=\frac{1}{3}\left[2\left(A^{2}-3 B\right)^{0.5} * \cos \left(\theta_{2}\right)+A\right]$

where $\theta_{2}=\theta_{1}+120$

$X_{3}=\frac{1}{3}\left[2\left(A^{2}-3 B\right)^{0.5} * \cos \left(\theta_{3}\right)+A\right]$

where $\theta_{3}=\theta_{1}+240$

And recognized on naming constants $(\mathrm{A}, \mathrm{B}, \mathrm{C})$ in the flexibility and plasticity, vibrations and mechanics of fluids letters $\left(\mathrm{I}_{1}, \mathrm{I}_{2}, \mathrm{I}_{3}\right)$ as, respectively, and this label came from an Invariants, and it means constants that do not change or constants stress that do not change stress invariants. There are certain constants, whatever the label $(\mathrm{A}, \mathrm{B}, \mathrm{C})$ or $\left(\mathrm{I}_{1}, \mathrm{I}_{2}, \mathrm{I}_{3}\right)$ In the case of recycling extended stress on one axes in a certain direction. The values of nine for this period will be changed, but these constants will be the same for the new extended matrix or does not change, hence the label or coding $\left(\mathrm{I}_{1}, \mathrm{I}_{2}, \mathrm{I}_{3}\right)$ instead of (A, B, C).

Thus, samples can be circulated depending on what the user in engineering books for flexibility and plasticity, vibrations and fluid mechanics, as follows:

$$
+\lambda^{3}-\mathbf{I}_{1} \lambda^{2}+\mathbf{I}_{2} \lambda-\mathbf{I}_{3}=\mathbf{0}
$$

$\mathrm{I}_{1}=\Sigma \mathrm{T}_{\mathrm{ii}}=\operatorname{trace}\left[\mathrm{T}_{\mathrm{ii}}\right], \mathrm{I}_{2}=\Sigma \operatorname{Miner}\left[\mathrm{T}_{\mathrm{ii}}\right], \mathrm{I}_{3}=\operatorname{determent}[\mathrm{T}]$

\section{Comparison between Classic and modern methods}

The first test: Solving the cubic equation in two ways (a) the Classic Method (b) the New Method:

$$
2 \lambda^{3}-\lambda^{2}-2 * \lambda+1=0
$$

\section{a. Classic Method}

$$
\begin{aligned}
& 2 \lambda^{3}-\lambda^{2}-2 \lambda+1=0 \quad \text { Let } \lambda=1 \\
& \Rightarrow(2)(1)^{3}-(1)^{2}-2(1)+1=0 \quad O K
\end{aligned}
$$

The fact that the first hypothesis $\lambda=1$ is not always easy because it comes from several attempts which did not achieve the equation to that we get to try investigating the equation if the assumption that the figure that achieves the equation is (9).

Here, we need (18) attempts negative

$$
\begin{aligned}
& \lambda - 1 \longdiv { 2 \lambda ^ { 3 } - \lambda ^ { 2 } - 2 \lambda + 1 } \\
& \text { ح } 2 \frac{ \pm 2 \lambda^{3} \pm 2 \lambda^{2}}{+\lambda^{2}-2 \lambda+1} \\
& \text { ح } 2 \text { بالطر } \pm \lambda^{2} \pm \lambda \\
& \text { - } \lambda+1 \\
& \tau \frac{ \pm \lambda \pm 1}{00}
\end{aligned}
$$

and positive until this figure is captured, which achieves the equation and naturally depends on the experience of the professor.

$$
\begin{aligned}
& \therefore \frac{2 \lambda^{3}-\lambda^{2}-2 \lambda+1}{\lambda-1}=2 \lambda^{2}+\lambda-1 \\
& \therefore 2 \lambda^{2}+\lambda-1=0 \Rightarrow(2 \lambda-1)(\lambda+1)=0 \\
& \therefore \frac{2 \lambda^{3}-\lambda^{2}-2 \lambda+1}{\lambda-1}=2 \lambda^{2}+\lambda-1 \\
& \therefore 2 \lambda^{2}+\lambda-1=0 \Rightarrow(2 \lambda-1)(\lambda+1)=0 \\
& 2 \lambda=1 \Rightarrow \lambda=\frac{1}{2} \text { and } \lambda=-1 \\
& \therefore \lambda_{1}=1 ; \lambda_{2}=0.5 ; \lambda_{3}=-1
\end{aligned}
$$

\section{b. New Method}

The cubic equation is : $2 \lambda^{3}-\lambda^{2}-2 * \lambda+1=0 \quad(\div 2)$

then: $\lambda^{3}-0.5 * \lambda^{2}-\lambda+0.5=0$

$\because \mathrm{I}_{1}=0.5, \mathrm{I}_{2}=-1$ and $\mathrm{I}_{3}=-0.5$

$$
\begin{aligned}
& \therefore \theta=\cos ^{-1}\left[\frac{I_{1}^{3}-4.5 I_{1} I_{2}+13.5 I_{3}}{\left(I_{1}^{2}-3 I_{2}\right)^{1.5}}\right] \Rightarrow \\
& \theta=\cos ^{-1}\left[\frac{(0.5)^{3}-4.5(0.5)(-1)+13.5(-0.5)}{\left((0.5)^{2}-3(-1)\right)^{1.5}}\right] \\
& \theta=\cos ^{-1}\left[\frac{-4.375}{5.86}\right]=\cos ^{-1}[-0.75]=138.3^{\circ} \\
& \theta=138.3 \Rightarrow \theta_{1}=\frac{\theta}{3}=\frac{138.3}{3}=46.1^{\circ} \\
& \text { and } \\
& \text { also } \theta_{3}=\theta_{1}+240=60+240=286.1^{\circ} \\
& \therefore \lambda_{1}=\frac{1}{3}\left(2 *\left(\mathrm{I}_{1}{ }^{2}-3 \mathrm{I}_{2}\right)^{0.5} * \cos \left(\theta_{1}\right)+I_{1}\right) \\
& =\frac{1}{3}\left(2 *\left(0.5^{2}-3(-1)\right)^{0.5} * \cos (46.1)-1\right)=1 \\
& \therefore \lambda_{2}=\frac{1}{3}\left(2 *\left(\mathrm{I}_{1}{ }^{2}-3 \mathrm{I}_{2}\right)^{0.5} * \cos \left(\theta_{2}\right)+I_{1}\right) \\
& =\frac{1}{3}\left(2 *\left(0.5^{2}-3(-1)\right)^{0.5} * \cos (166.1)-1\right)=-1 \\
& \therefore \lambda_{3}=\frac{1}{3}\left(2 *\left(\mathrm{I}_{1}{ }^{2}-3 \mathrm{I}_{2}\right)^{0.5} * \cos \left(\theta_{3}\right)+I_{1}\right) \\
& =\frac{1}{3}\left(2 *\left(0.5^{2}-3(-1)\right)^{0.5} * \cos (286.1)-1\right)=0.5 \\
& W
\end{aligned}
$$

Where $\lambda_{1}>\lambda_{2}>\lambda_{3}$ then the principal stresses (eigen values ) are $: \lambda_{1}=1 \quad ; \lambda_{2}=0.5 \quad ; \lambda_{3}=-1$ 


\section{Practical applications}

\subsection{In Elasticity}

If the point $(5,3,0)$ verifies the equation, find the major stresses (eigenvalues) for a Stress Tensor at this point.

$$
[\sigma]=\left[\begin{array}{ccc}
1.5 x_{1}^{2} x_{2}^{2} & -x_{1} x_{2}^{3} & 0 \\
-x_{1} x_{2}^{3} & 0.25 x_{2}^{4} & 0 \\
0 & 0 & 100
\end{array}\right]
$$

When $\left(\mathrm{I}_{1}, \mathrm{I}_{2}, \mathrm{I}_{3}\right)$ are the stress invariants at point $(5,3,0)$ :

$$
[\sigma]=\left[\begin{array}{ccc}
1.5 x_{1}^{2} x_{2}^{2} & -x_{1} x_{2}^{3} & 0 \\
-x_{1} x_{2}^{3} & 0.25 x_{2}^{4} & 0 \\
0 & 0 & 100
\end{array}\right]=\left[\begin{array}{ccc}
337.5 & -135 & 0 \\
-135 & 20.25 & 0 \\
0 & 0 & 100
\end{array}\right] M p a
$$

Then : $\mathrm{I}_{1}=\Sigma\left[\sigma_{\mathrm{ii}}\right]=457.75, \mathrm{I}_{2}=\Sigma$ miner $\left[\sigma_{\mathrm{ii}}\right]=24384.375$, $\mathrm{I}_{3}=\operatorname{det} \cdot[\sigma]=-1139062.5$.

$\therefore \sigma^{3}-457.75 \sigma^{2}+24384.375 \sigma+1139062.5=0$

$$
\therefore \theta=\cos ^{-1}\left[\frac{I_{1}^{3}-4.5 I_{1} I_{2}+13.5 I_{3}}{\left(I_{1}^{2}-3 I_{2}\right)^{1.5}}\right]=53.0033^{\circ}
$$

$\sigma_{1}=\frac{1}{3}\left[\left(2 \sqrt{I_{1}^{2}-3 I_{2}}\right) \cos \theta_{1}+I_{1}\right]=387.1702 \mathrm{Mpa}$ $\sigma_{2}=\frac{1}{3}\left[\left(2 \sqrt{I_{1}^{2}-3 I_{2}}\right) \cos \theta_{2}+I_{1}\right]=-29.4202 \mathrm{Mpa}$ $\sigma_{3}=\frac{1}{3}\left[\left(2 \sqrt{I_{1}^{2}-3 I_{2}}\right) \cos \theta_{3}+I_{1}\right]=100 \mathrm{Mpa}$

$\sigma_{1}=387.1702 \mathrm{Mpa}, \quad \sigma_{2}=100 \mathrm{Mpa}, \quad \sigma_{3}=-29.4202 \mathrm{Mpa}$ Where: $\sigma_{1}>\sigma_{2}>\sigma_{3}$

\subsection{In Plasticity}

The values given below for the strains to the point in the three-axis system, to find eigenvalues for Strain Tensor.

$$
[\varepsilon]=\left[\begin{array}{ccc}
261 & 175.5 & 65 \\
175.5 & -261 & 0 \\
65 & 0 & 198
\end{array}\right] \mu \text { strain }
$$

where:

$$
\begin{gathered}
I_{1}=\sum \varepsilon_{i i}=\operatorname{trace}\left(\varepsilon_{i j}\right)=198 \\
I_{2}=\sum \operatorname{Miner}\left(\varepsilon_{i i}\right)=-103146.3 \\
I_{3}=\operatorname{det}\left[\varepsilon_{i j}\right]=-1.843 \times 107 \\
\therefore \varepsilon^{3}-I_{1} \varepsilon^{2}+I_{2} \varepsilon-I_{3}=0 \Rightarrow \\
\varepsilon^{3}-198 \varepsilon^{2}-103146.3 \varepsilon+1.843 \times 10^{7}=0 \\
\therefore \theta=\cos ^{-1}\left[\frac{I_{1}^{3}-4.5 I_{1} I_{2}+13.5 I_{3}}{\left(I_{1}^{2}-3 I_{2}\right)^{1.5}}\right]=136.6994^{\circ} \\
\theta=136.6994 \Rightarrow \theta_{1}=45.5614, \quad \theta_{2}=165.566 \quad \theta_{3}=285.566
\end{gathered}
$$

$\varepsilon_{1}=\frac{1}{3}\left[\left(2 \sqrt{I_{1}^{2}-3 I_{2}}\right) \cos \theta_{1}+I_{1}\right]=341.5$ ustrain

$\varepsilon_{2}=\frac{1}{3}\left[\left(2 \sqrt{I_{1}^{2}-3 I_{2}}\right) \cos \theta_{2}+I_{1}\right]=-315.2$ ustrain

$\varepsilon_{3}=\frac{1}{3}\left[\left(2 \sqrt{I_{1}{ }^{2}-3 I_{2}}\right) \cos \theta_{3}+I_{1}\right]=172.4$ ustrain

$\therefore \varepsilon_{1}=341.5$ ustrain, $\varepsilon_{2}=172.4$ ustrain, $\varepsilon_{3}=-315.2$ ustrain where : $\varepsilon_{1}>\varepsilon_{2}>\varepsilon_{3}$

\subsection{In Vibrations}

For the spring-mass system, find the natural frequency of this system: -

$$
\begin{aligned}
& {\left[\begin{array}{l}
x_{1} \\
x_{2} \\
x_{3}
\end{array}\right]=\frac{w^{2} m}{k}\left[\begin{array}{lll}
\frac{4}{3} & \frac{2}{3} & \frac{1}{3} \\
\frac{4}{3} & \frac{8}{3} & \frac{4}{3} \\
\frac{4}{3} & \frac{8}{3} & \frac{7}{3}
\end{array}\right] *\left[\begin{array}{l}
x_{1} \\
x_{2} \\
x_{3}
\end{array}\right]} \\
& {\left[\begin{array}{l}
x_{1} \\
x_{2} \\
x_{3}
\end{array}\right]=\frac{w^{2} m}{3 k}\left[\begin{array}{lll}
4 & 2 & 1 \\
4 & 8 & 4 \\
4 & 8 & 7
\end{array}\right] *\left[\begin{array}{l}
x_{1} \\
x_{2} \\
x_{3}
\end{array}\right] \Rightarrow} \\
& T=\frac{w^{2} m}{3 k}\left[\begin{array}{lll}
4 & 2 & 1 \\
4 & 8 & 4 \\
4 & 8 & 7
\end{array}\right] \Rightarrow T=\lambda\left[\begin{array}{lll}
4 & 2 & 1 \\
4 & 8 & 4 \\
4 & 8 & 7
\end{array}\right]
\end{aligned}
$$

To facilitate a solution, we can impose :

$$
\lambda_{i}=\frac{w^{2} m}{3 k} \therefore w^{2}=\frac{3 k}{\lambda_{i} m} \Rightarrow w_{i}=\sqrt{\frac{3 k}{\lambda_{i} m}}
$$

First: - calculate the constants:

$$
I_{1}=19 \quad, \quad I_{2}=72 \quad, \quad I_{3}=72
$$

the general formula of the cubic equation is: -

$$
\lambda^{3}-I_{1} \lambda^{2}+I_{2} \lambda-I_{3}=0 \Rightarrow \lambda^{3}-19 \lambda^{2}+72 \lambda-72=0
$$

Using the equation first, second and fourth we get: -

$$
\begin{gathered}
\therefore \theta=\cos ^{-1}\left[\frac{I_{1}^{3}-4.5 I_{1} I_{2}+13.5 I_{3}}{\left(I_{1}{ }^{2}-3 I_{2}\right)^{1.5}}\right]=16.43^{\circ} \\
\therefore \theta=16.43, \quad \theta_{1}=5.48, \quad \theta_{2}=125.48, \quad \theta_{3}=245.48
\end{gathered}
$$

$$
\begin{aligned}
& \lambda_{1}=\frac{1}{3}\left[\left(2 \sqrt{I_{1}^{2}-3 I_{2}}\right) \cos \theta_{1}+I_{1}\right]=14.33 \\
& \lambda_{2}=\frac{1}{3}\left[\left(2 \sqrt{I_{1}^{2}-3 I_{2}}\right) \cos \theta_{2}+I_{1}\right]=1.68 \\
& \lambda_{3}=\frac{1}{3}\left[\left(2 \sqrt{I_{1}^{2}-3 I_{2}}\right) \cos \theta_{3}+I_{1}\right]=3
\end{aligned}
$$




$$
\begin{gathered}
\lambda_{1}=14.33 \Rightarrow w_{1}=0.457 \sqrt{\mathrm{k} / \mathrm{m}} \mathrm{rad} / \mathrm{sec} \\
\lambda_{2}=3.00 \Rightarrow w_{2}=1.00 \sqrt{\mathrm{k} / \mathrm{m}} \mathrm{rad} / \mathrm{sec} \\
\lambda_{3}=1.68 \Rightarrow w_{3}=1.34 \sqrt{\mathrm{k} / \mathrm{m}} \mathrm{rad} / \mathrm{sec} \\
\text { where } \lambda_{1}>\lambda_{2}>\lambda_{3} \text { or } \omega_{3}>\omega_{2}>\omega_{1}
\end{gathered}
$$

\subsection{In fluid mechanics:}

The main stresses (eigenvalues) are found out to the following compounds of stress.

$[\sigma]=\left[\begin{array}{ccc}36 & 27 & 10 \\ 27 & -36 & 0 \\ 16 & 0 & 18\end{array}\right] M P a$

where:

$\mathrm{I}_{1}=\mathrm{A}=\Sigma\left[\sigma_{\mathrm{ii}}\right]=18$

$\mathrm{I}_{2}=\mathrm{B}=\Sigma$ miner $\left[\sigma_{\mathrm{ii}}\right]=-2125$

$\mathrm{I}_{3}=\mathrm{C}=\operatorname{det}[\sigma]=-32850$

$\therefore \theta=\cos ^{-1}\left[\frac{I_{1}^{3}-4.5 I_{1} I_{2}+13.5 I_{3}}{\left(I_{1}^{2}-3 I_{2}\right)^{1.5}}\right]=119^{\circ}$

$\therefore \theta=119^{\circ}, \quad \theta_{1}=39.66^{\circ}, \quad \theta_{2}=159.66^{\circ}, \quad \theta_{3}=279.66^{\circ}$

$\sigma_{1}=\frac{1}{3}\left[\left(2 \sqrt{I_{1}^{2}-3 I_{2}}\right) \cos \theta_{1}+I_{1}\right]=48 M P a$

$\sigma_{2}=\frac{1}{3}\left[\left(2 \sqrt{I_{1}^{2}-3 I_{2}}\right) \cos \theta_{2}+I_{1}\right]=-45.161 M P a$

$\sigma_{3}=\frac{1}{3}\left[\left(2 \sqrt{I_{1}^{2}-3 I_{2}}\right) \cos \theta_{3}+I_{1}\right]=15.1511 M P a$

where:

$\therefore \sigma_{1}=48 \mathrm{MPa}, \quad \sigma_{2}=15.1511 \mathrm{MPa}, \quad \sigma_{3}=-45.161 \mathrm{MPa}$

\subsection{In mathematical.}

To find the general solution of the system follows: -

$\frac{d x}{d t}+5 x+7 y-5 z=0 \quad \frac{d y}{d x}+4 y-z=0, \quad \frac{d z}{d t}+2 x+8 y-3 z=0$

This system can be written as an equation differential form of the first rank matrices:

$\frac{d u}{d t}+M u=0 \Rightarrow M=\left[\begin{array}{lll}5 & 7 & -5 \\ 0 & 4 & -1 \\ 2 & 8 & -3\end{array}\right] \& u=\left[\begin{array}{l}x \\ y \\ z\end{array}\right]$

The general equation for such a situation is: where (V) is a vector of three dimensions of vertical fixed

\section{Now we find the constants of cubic equation:}

$$
\begin{gathered}
I_{1}=\sum\left[M_{i i}\right]=5+4-3=6 \quad, \quad I_{2}=\sum \operatorname{Miner}\left[M_{i i}\right]=11, \\
I_{3}=\operatorname{det}[M]=6 \\
\therefore \lambda^{3}-I_{1} \lambda^{2}+I_{2} \lambda-I_{3}=0 \rightarrow \lambda^{3}-6 \lambda^{2}+11 \lambda-6=0
\end{gathered}
$$

Applying the equations first, second and fourth we get:

$$
\begin{aligned}
& \therefore \theta=\cos ^{-1}\left[\frac{I_{1}^{3}-4.5 I_{1} I_{2}+13.5 I_{3}}{\left(I_{1}^{2}-3 I_{2}\right)^{1.5}}\right]=90^{\circ} \\
& \theta=90^{\circ} \therefore \theta_{1}=30^{\circ}, \theta_{2}=150^{\circ}, \theta_{3}=270^{\circ} \\
& \therefore \lambda_{1}=\frac{1}{3}\left(2 \sqrt{\left.\left(I_{1}-3 I_{2}\right) * \cos \theta_{1}+I_{1}\right)=3}\right. \\
& \lambda_{2}=\frac{1}{3}\left(2 \sqrt{\left(I_{1}-3 I_{2}\right)} * \cos \theta_{2}+I_{1}\right)=1 \\
& \lambda 3=\frac{1}{3}\left(2 \sqrt{\left(I_{1}-3 I_{2}\right)} * \cos \theta_{3}+I_{1}\right)=2 \\
& \lambda_{1}=3 \quad, \quad \lambda_{2}=1 \quad \& \quad \lambda_{3}=2 \\
& \text { or } \lambda_{1}=3, \quad \lambda_{2}=2, \quad \lambda_{3}=1
\end{aligned}
$$

Since the eigenvectors are: -

$$
\begin{gathered}
\therefore V_{1}=\left[\begin{array}{c}
-1 \\
1 \\
1
\end{array}\right], \quad V_{2}=\left[\begin{array}{l}
1 \\
1 \\
2
\end{array}\right], \quad V_{3}=\left[\begin{array}{l}
2 \\
1 \\
3
\end{array}\right] \quad \therefore u=V e^{\lambda t} \\
\therefore u_{1}=\left(\begin{array}{c}
-1 \\
1 \\
1
\end{array}\right) * e^{3 t}, \quad u_{2}=\left(\begin{array}{l}
1 \\
1 \\
2
\end{array}\right) * e^{2 t} \& u_{3}=\left(\begin{array}{l}
2 \\
1 \\
3
\end{array}\right) * e^{t}
\end{gathered}
$$

General solution is:

$$
\therefore u=c_{1} u_{1}+c_{2} u_{2}+c_{3} u_{3}=c_{1}\left(\begin{array}{c}
-1 \\
1 \\
1
\end{array}\right) e^{3 t}+c_{2}\left(\begin{array}{l}
2 \\
1 \\
3
\end{array}\right) e^{t}+c_{3}\left(\begin{array}{l}
1 \\
1 \\
2
\end{array}\right) e^{2 t}
$$

The general solution can be written as a mathematical formula rather than engineering as follows: -

$$
\because u=\left\{\begin{array}{l}
x \\
y \\
z
\end{array}\right\} \Rightarrow \quad \begin{aligned}
& x \\
& y=-c_{1} e^{3 t}+2 c_{2} e^{t}+c_{3} e^{2 t}+c_{2} e^{t}+c_{3} e^{2 t} \\
& z=c_{1} e^{3 t}+3 c_{2} e^{t}+2 c_{3} e^{2 t}
\end{aligned}
$$

\section{Conclusion}

1- The roots of the cubic equation (or matrix extended) is found as follows:

New Equation for Eigen Values:

$\lambda_{i}=\frac{1}{3}\left[\left(2 \sqrt{I_{1}^{2}-3 I_{2}}\right) \cos \theta_{i}+I_{1}\right]$

2 - The angle is calculated from the following equation: The Principal Angle equation:

$$
\theta=\cos ^{-1}\left[\frac{I_{1}^{3}-4.5 I_{1} I_{2}+13.5 I_{3}}{\left(I_{1}^{2}-3 I_{2}\right)^{1.5}}\right]
$$

3- Three angles are identified by which three roots can be determined as follows:

The three angles and three roots of equations $\left(\lambda_{\mathrm{i}}\right)$ :

$\lambda_{1}$ at $\theta_{1}=\theta / 3$

$\lambda_{2}$ at $\theta_{2}=\theta / 3+120$

$\lambda_{3}$ at $\theta_{3}=\theta / 3+240$ 


$$
\begin{aligned}
& \lambda_{1}=\frac{1}{3}\left[\left(2 \sqrt{I_{1}^{2}-3 I_{2}}\right) \cos \theta_{1}+I_{1}\right] \\
& \lambda_{2}=\frac{1}{3}\left[\left(2 \sqrt{I_{1}^{2}-3 I_{2}}\right) \cos \theta_{2}+I_{1}\right] \\
& \lambda_{3}=\frac{1}{3}\left[\left(2 \sqrt{I_{1}^{2}-3 I_{2}}\right) \cos \theta_{3}+I_{1}\right]
\end{aligned}
$$

4- The constants that do not change (Invariants) is calculated from the equations below. It can be seen the following things about these constants $\left(\mathrm{I}_{1}, \mathrm{I}_{2}, \mathrm{I}_{3}\right)$ as follows: -

a-These constants are not changing, if not changed, because the roots have been calculated at $(\lambda \mathrm{i})$ at any root of this equation.

b- Noting that $\left(\mathrm{I}_{1}\right)$ means the algebraic sum of the elements of the diameter of the matrix $[\mathrm{T}], \mathbf{I}_{\mathbf{1}}=\mathbf{\Sigma} \mathbf{T}_{\mathrm{ii}}=\operatorname{trace}\left[\mathbf{T}_{\mathrm{ii}}\right]$.

c-Noting that (I2) means the algebraic sum (Miner) to the diameter only elements of the matrix [T], $\mathrm{I} 2=\Sigma$ Miner [Tii ]. d-Noting that $\left(\mathrm{I}_{3}\right)$ means a specific matrix $[\mathrm{T}], \mathbf{I}_{\mathbf{3}}=\operatorname{det}$. $\left[\mathbf{T}_{\mathbf{i j}}\right]$.

\section{REFERENCES}

[1] R. Kumar and V. Kumar, Study on the Evolution of Cubic Equation Solving. Rep Opinion 2020;12(4):1-6. doi:10.7537/marsroj120420.01. [2] E. L. Karunia, S. P. Udjianna , W. I. Sapto and G. G. Hanni, Generating roots of cubic polynomials by Cardano's approach on correspondence analysis, Heliyon, V.6(6) Jun 2020.

[3] A. T. Tiruneh, A simplified expression for the solution of cubic polynomial equations using function evaluation, arXiv:2002.06976v1 [math.GM] 14 Feb 2020.

[4] A. H. Salas and S. C. Trujillo, A New Approach for Solving the Complex Cubic-Quantic Duffing Oscillator Equation for Given Arbitrary Initial Conditions, Mathematical Problems in Engineering, V.398(975) 2020, Article ID 3985975,1- 8.

[5] R. E. Ghali, M. A. Almahalebi and S. K. Kabbaj, General solution and hyperstability results for a cubic radical functional equation related to quadratic mapping, Proyecciones, vol.39(1) Feb. 2020, 107-122 .

[6] I. C. Javier, The numerical problems within analytical methods of solution for cubic equations of state, Ingeniería y Competitividad, V.14(1) (2012)75 - 89.

[7] P. Shouthiri and S. Disne, Solution to Cubic Equation using Java Programming", European Journal of Mathematics and Computer Science, V. 7 (1), 2020.

[8] W. A. Gao , H. F. Ismael, H. M. Ahmad , B. A. Hasan and H. M Baskonus, Optical Soliton Solutions of the Cubic-Quartic Nonlinear Schrödinger and Resonant Nonlinear Schrödinger Equation with the Parabolic Law, Appl. Sci. 219(10)2020; doi:10.3390/app10010219.

[9] V. Govindan,C. Park, S. Pinelas \& S. Baskaran, Solution of a 3-D cubic functional equation and its stability. AIMS Mathematics, 5(3)2020, 1693-1705. https://doi.org/10.3934/math.2020114.

[10] S. R. Javier, The Midpoints Between Roots Reveal the Quartic Equation, The American Mathematical Monthly, 127(3)2020, 258-262, DOI:10.1080/00029890.2020.1697589.

[11] D. A. Abdullah, MATLAB and Engineering Applications, third Edition, 2019. 